\title{
Autonomous Cooperating Processes for the Improvement of Food Quality
}

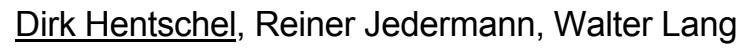 \\ IMSAS (Institute for Microsensors, -actuators and -systems), University of Bremen FB1, \\ Otto-Hahn-Allee NW1, 28359 Bremen, Germany \\ IMSAS is part of the Microsystems Center Bremen (MCB) \\ Phone: +49 (421) 218-3586, fax: +49 (421) 218-4774, dhentschel@imsas.uni-bremen.de
}

\begin{abstract}
In most cases, logistical planning tasks are a complex problem that is difficult to solve because of dynamic influences that cannot be foreseen. The concept of autonomous cooperating processes is an approach whereby the logistical objects themselves solve those tasks. Each object in the transport chain (freight, means of transport, warehouse...) is represented by a separate independent software unit (a so-called agent). These agents can interact and negotiate with each other. Each agent looks for a partial solution that is beneficial to the local object. In our approach, we put a focus on freight agents that observe the conditions during the process of transportation. Based on the evaluation of temperature measurement data, which are derived from a wireless sensor network inside the cargo hold, the remaining days of acceptable quality are predicted. In the case of an interruption of the cold chain, the agents can send warning messages to the freight owner or even make decisions on their own (e.g. a change to the planned route) to optimize the quality of the freight items on their arrival.
\end{abstract}

\section{Introduction}

In the past years, the aspect of food quality has become more important to the customers. The acceptance limits are stricter in view to firmness, colour and taste. The demand for food in the best possible quality state at the best possible price has grown. Much effort has been made in the research sector to fulfil the consumer's wishes. A closed cold chain is essential to achieve optimal quality at the end of the transportation from farm to fork.

There are already solutions available on the market to observe transportation processes. Modern transport vehicles are equipped with a telematic system that transmits the present GPS coordinates at fixed time intervals to a central server, so the freight owner can track the transportation route. For freight items that need special transportation conditions like cooling, those systems can be extended with a temperature sensor. To ensure a closed cold chain, and for reasons of transparency, the measured temperature is transmitted, too. However, with only one sensor, spatial temperature deviations cannot be considered, but experiments have shown that the temperature inside the cargo hold varies in a noticeable way, so the quality of fruit that is transported together is not equal.

An increase in the number of sensors is necessary to monitor these deviations of temperature. However, this is accompanied by an increase in the amount of data that has to be transmitted. For the transmission, public cellular phone networks are used. The transmission of data in those networks is usually charged by the amount of data. Hence, this approach causes rising costs. Another disadvantage exists in view of the interpretation of the data for the freight owner. The assignment of sensor data to the freight and the meaning of a detected temperature deviation are difficult. This does not increase the transparency but rather causes confusion. In our approach, we present a tracking solution without those disadvantages. We implemented a local pre-processing of the sensor data. A wireless sensor network with a number of battery-powered sensor nodes is installed in the cargo hold. Each sensor node is equipped with a so-called IEEE 802.15.4 interface that allows it to send the measured data to a local gateway that is also installed in the vehicle. This gateway unit has enough computation power to pre-process all the incoming data. An intelligent assignment of

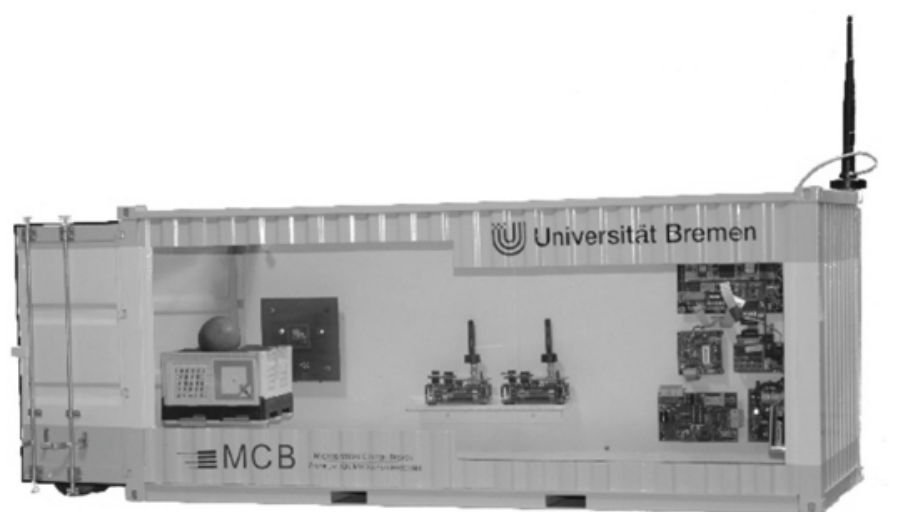

Figure 1: model of intelligent container, scaled 1:8 
measured values to the freight items is performed with an RFID solution. Each freight item that enters or leaves the cargo hold is identified by a reader unit that is mounted at the door. An individual temperature can be assigned to each freight item. Based on that data, a prediction of the quality state is calculated, so the notification to the mentioned external server can be very concise.

We call this concept the "intelligent container" [1]. We have already implemented a 1:8 scaled model, which is shown in Figure 1. However, this concept is not limited to containers: it can be used in any transport situation where a supervision of the quality is required. The core ideas of our concept are explained in detail in the following sections.

\section{Shelf-life modelling}

To make a prediction of the food quality, we use a model that permits us to draw a conclusion from a given temperature history to the current state of quality. For each kind of fruit, an optimal temperature range for storage and transportation exists. If the temperature is higher, the process of decay will be accellerated. However, if the temperature is too low, the quality will also be decreased because of chilling injuries.

For a mathematical description of the decay effect, we use the law of Svante Arrhenius for reaction kinetics [2]:

$$
k=k_{\text {ref }} \cdot e^{\frac{E_{\mathrm{a}}}{R} \cdot\left(\frac{1}{T_{\mathrm{ref}}}-\frac{1}{T}\right)} .
$$

In this equation, $k$ is the reaction rate at the temperature $T$, whereas $k_{\text {ref }}$ is the reaction rate at the reference temperature $T_{\text {ref. }} E_{\mathrm{a}}$ is the activation energy, measured in $\mathrm{J} / \mathrm{mol}$. $R$ is the gas constant: $R=8.314 \mathrm{~J} /(\mathrm{mol} \cdot \mathrm{K})$. The quality can be described with the next equation [3]:

$$
Q=Q_{0} \cdot e^{-k \cdot t} \text {. }
$$

In this equation, $Q$ is the quality at time $t$ and $Q_{0}$ is the initial value for quality. Based on the equations (1) and (2), we can calculate the keeping quality $K Q$ with:

$$
K Q=\frac{\log _{e}\left(\frac{Q_{0}}{Q_{1}}\right)}{k},
$$

where $Q_{1}$ is the acceptance limit. $K Q$ is a time value. It states for how many days the quality will be in an acceptable range. For a better fit of the functions to the realistic physical behaviour and to constitute the effects of chilling injuries in addition to the decay acceleration, we developed an equation to calculate the loss per day $L$, which is a function of the current temperature $T$ :

$$
L(T)=\frac{k_{1}(T)+k_{2}(T)}{k_{1}\left(T_{s}\right)+k_{2}\left(T_{s}\right)}
$$

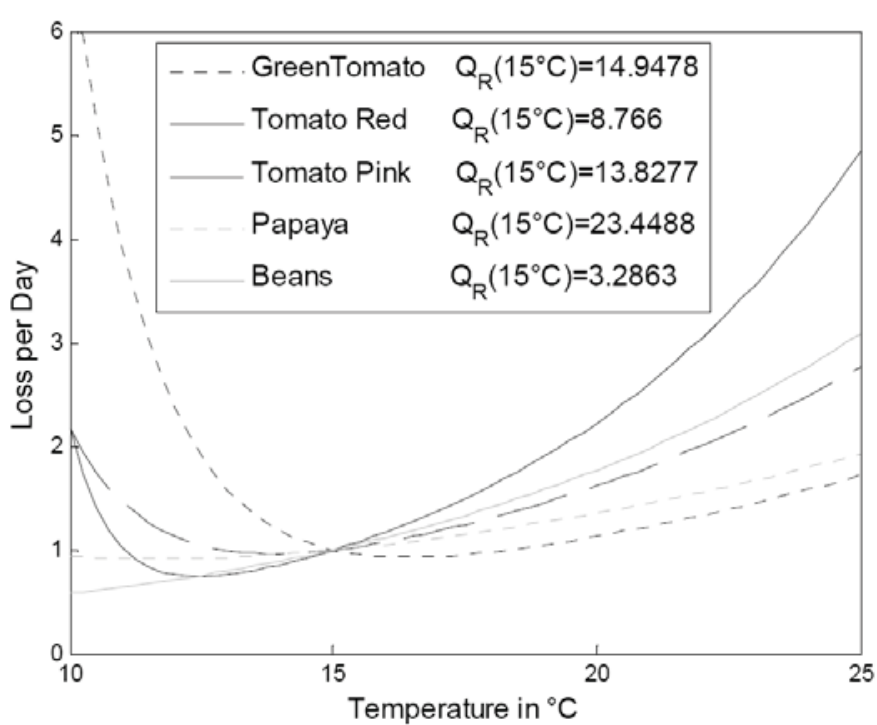

Figure 2: Loss of shelf life per day for different kinds of fruits
For $k_{1}$ and $k_{2}$, the $k$ of equation (1) is inserted with different values for $k_{\text {ref, }}, T_{\text {ref }}$ and $E_{\mathrm{a}}$. These parameters can be determined by experiments under laboratory conditions $[3,4] . T_{\mathrm{s}}$ is the final storage temperature.

In Figure 2, we present some examples of shelf-life loss plotted for different kinds of fruit, where $Q_{R}$ is the quality at a reference temperature of $15{ }^{\circ} \mathrm{C}$. There is an individual optimum for each kind of fruit. For lower temperatures, the chilling injuries come into effect, whereas for higher temperatures the decay occurs. For the combined transport of different kinds of fruit, it should be considered that each kind has an individual reaction to these temperature effects. 


\section{Shelf-life prediction by intelligent software agents}

In our demonstration scenario of the intelligent container [1], the intelligence of the vehicle is implemented by using software agents that represent the freight items. Software agents are small independent computer programs, which run on a distributed platform. In our case, this is the processor platform inside the vehicle. We use the JADE (Java Agent DEvelopment) framework to run the agents [5]. The major advantage of this approach is the ability of software agents to be transferred to another platform. Hence, it is possible to have a parallel transit of agents and freight items: in parallel to each loading and unloading process of freight items, the agents are transmitted, too. For example, during an unloading process of a delivery truck to a warehouse, the associated software agent would be transmitted from the vehicle's platform to the platform of the warehouse. So, the agent is always near its represented object.

In our concept, the processor platform is connected to a wireless sensor network, which measures the environmental storage conditions at different points inside the cargo hold. The platform is also connected to an RFID reader, which is mounted next to the door. During the loading and unloading processes, the RFID reader identifies the pallets that enter or leave the transport vehicle. Hence, the processor platform always knows which freight is inside the cargo hold. If the associated software agent has not yet been transferred, it will be queried from the other platform through a WLAN network. This concept is described in detail in [6].

During transportation, the wireless sensor network transfers the measured data to the processor platform of the vehicle. The agents pre-process the incoming data by comparing them with the recommended transport conditions, which are stored individually for each freight object as a data record inside its associated software agent.

Because of the pre-processing of the measured data in our concept, it is not necessary to send any data from the internal sensors to the external server system. Only in the case of danger to the freight is a warning message generated and sent through the cellular network. The base for that warning message is the quality indicator, which was described in the previous section. This approach decreases significantly the amount of data, since it is assumed that the values are usually within the given limits. Further decisions based on this quality indicator are described in the following section.

\section{Decision freedom}

By allowing the agents to make decisions on their own, the described system can be extended to more autonomy. If the deviation from the optimal values exceeds a given limit, the agent can perform an action. All the actions fall into one of the four different classes [7]: "no freedom", "drawing conclusions", "adjust solution" and "adjust goal", cf. Table 1.

\begin{tabular}{|c|c|c|c|}
\hline Degree & Class & Decision scope & Example \\
\hline 0 & No freedom & None & Decisions are made centrally. \\
\hline 1 & $\begin{array}{l}\text { Drawing } \\
\text { conclusions }\end{array}$ & $\begin{array}{l}\text { Evaluation of } \\
\text { sensor data }\end{array}$ & $\begin{array}{l}\text { Object observes environmental conditions and } \\
\text { decides if they are a risk to quality. }\end{array}$ \\
\hline 2 & Adjust solution & Route planning & Transport route can be changed. \\
\hline 3 & Adjust goal & $\begin{array}{l}\text { Order } \\
\text { management }\end{array}$ & $\begin{array}{l}\text { Destination can be changed, based on } \\
\text { different orders. }\end{array}$ \\
\hline
\end{tabular}

Table 1: Degrees of decision freedom [7]

If the system has no freedom to decide at all, the decision scope of the agent is empty. This means that decisions are only made centrally and not individually by the objects (here: the vehicle). The second class allows the system to draw conclusions from given data. The decision scope consists of the evaluation of information provided by the local sensor network. The system checks if those values are inside defined limits and decides if that means a risk for the freight. This was described in the previous section.

The decision freedom in class 3 of Table 1 goes further. Based on the evaluation of the sensor data, the system can decide to change the originally planned solution to reach the goal. For a transport vehicle, the goal would be to deliver all the freight items in a proper state to their destinations. The solution is given by the route that has been planned before the beginning of the transportation process. For the agent, an action to adjust that solution could be to change the order of those destinations. We describe this approach in more detail in the next section.

Another way to adjust the solution, if a temperature deviation is detected, could be - for example - a change of the temperature set point of the cooling unit. However, this could cause chilling injuries to some freight, so the agent has to consider the consequences of this action. 


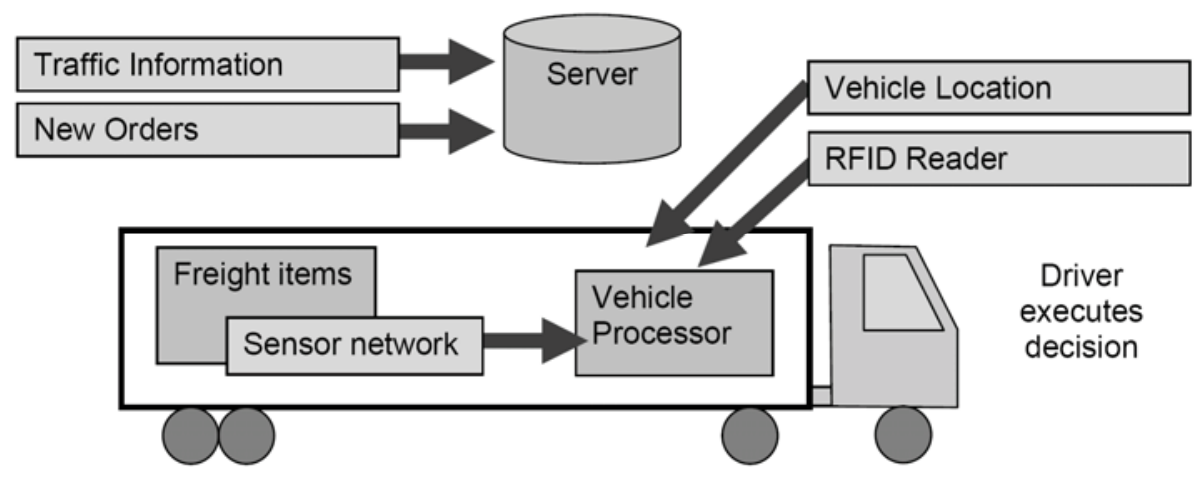

Figure 3: Communication path for dynamic route planning process

The highest class of decision freedom allows the goal to be adjusted. The information that is given by the ordering management system at the beginning of the transport can be changed. If the system reasons that the quality at the designated destination would be too low, it can assign a closer destination to that package. This would be carried out by contacting the order management server, which would make a suggestion of a different nearby customer to whom the package could also be delivered.

\section{Dynamic route-planning process}

In usual logistic processes, the degree of decision freedom of the freight is zero. No local decisions are allowed. We set up a concept that implements the second class of decision freedom (cf. Table 1). That means that we allow the system to change the planned solution. Also, we performed some experiments with software simulation to show the feasibility of this approach of autonomous control on the vehicle level.

In our concept, depicted in Figure 3, we have a delivery truck that distributes a number of packages from a storage centre to a number of different customers, towns or destinations: one package for each destination. Before the beginning of the tour, a multiple-vehicle optimization of all the available packages is made on a central server at the depot. The aim of this optimization is to assign similar driving times to each vehicle. Also, the predicted quality state of each package at the destination is considered.

Finally, at the beginning of the route, when the vehicles leave the depot, each truck has its own planned route to deliver those packages. The swapping of packages after beginning the tour is not possible. This planned route is stored in the vehicle. At each stop for delivery during the given route, the vehicle evaluates the quality state of its contained remaining goods. For the purpose of tracing and tracking the delivery, the vehicle sends the identification number (RFID) of the present delivered package and the location of the vehicle (GPS) to the central server. Further communication during the transportation is only necessary if a failure occurs.

The failures considered are an increase in the temperature that causes quality problems or a significant delay due to traffic congestion. In these cases, the vehicle has the ability to change the planned route. For that purpose, the vehicle sends a query to its server at the depot via mobile communication. It asks for a new route suggestion for the remaining destinations. The server has access to up-to-date information about the traffic situation, to geographic information, and to the position of the vehicle as well, but it has no access to the internal quality data of the truck.

To process this query, the server has to solve a travelling salesman problem (TSP). To save calculation time, we suppose a heuristic approach. That means a fast solution will be produced, which is not the optimal

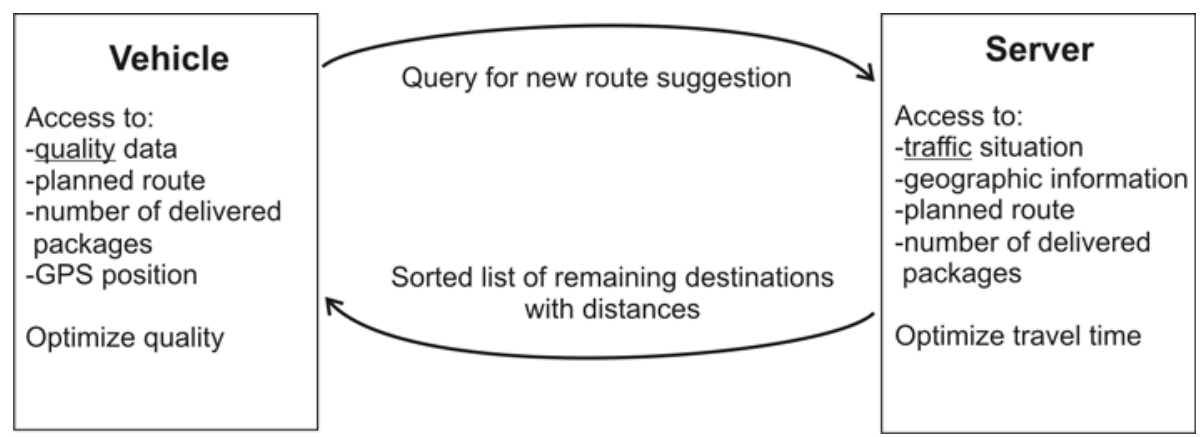

Figure 4: Interaction of vehicle and server 
solution, but a good one. An optimal solution to that problem would take hours or days of computation, which would not be acceptable during the transportation.

As a response to that query, the server sends a sorted list of the remaining towns, together with their distances. The list's order is optimized to travel time. This communication activity is depicted in Figure 4.

The elements of the received list are numbers, which refer to the original list. So, the amount of transmitted data is kept low (about 100 bytes). Another aspect is that these data are not usable by any other listeners on the communication path across public networks.

The list of towns can be transformed into a town circle by adding the current destination to the begin and to the end of the list as well. The system has the freedom to decide, where to start following the circle. Further, the direction of rotation has to be decided, e.g. clockwise or anticlockwise. We implemented an algorithm to find the pair of starting point and direction with the most benefits regarding the quality (priority) and travelling time. The quality is considered as the number of days of remaining shelf life.

\section{Software simulation}

To analyse the benefits of this approach in view of quality, we carried out some software simulations. The distribution truck is equipped with $N_{0}=20$ packages. The route is planned based on the shelf-life data of the loaded freight items. After the second delivery, we manipulate all the shelf-life data by subtracting a constant value. This value simulates a decrease in quality caused by a failure in the cold chain.

In Table 2, the results of 500 software experiments are shown. We compare four different strategies of reaction to that loss of quality. If the truck continues to follow the original route, without changing anything, on average 14.30 packages are delivered in time. The remaining shelf-life value has to be greater than zero. For 5.70 packages, this is not the case: they have no remaining shelf life at their destinations. This strategy is the worst case. So, we set the improvement to $0 \%$.

With the other three methods, we try to improve that number of delivered packages. The best possible method of improvement would be a full replanning. We save on average 2.11 additional packages. We set this improvement to $100 \%$. However, this takes a lot of calculation power, so it cannot be done by the processor inside the vehicle, so all the data for calculation have to be transferred to the central server. This means we need a large amount of data to be sent aross the mobile communication network from the vehicle to the server and back. This is accompanied by increasing costs for communication and, additionally, a loss of privacy. Hence, we prefer another approach.

\begin{tabular}{llll}
\hline Method & $\begin{array}{l}\text { Delivered } \\
\text { packages }\end{array}$ & Improvement & Driving time \\
\hline Continue with unchanged route & 14.30 & $0 \%$ & 74.68 hours \\
Local vehicle planning & 15.66 & $64.5 \%$ & 76.82 hours \\
Repeated vehicle planning & 15.75 & $68.6 \%$ & 75.80 hours \\
Full replanning (server) & 16.41 & $100 \%$ & 76.81 hours \\
\hline
\end{tabular}

Table 2: Comparison of different planning strategies (500 software experiments) [7]

As already described above, the vehicle's processor system is able to replan the route, just by requesting a suggested route from the server and considering the local data from the sensor network. On average, the number of delivered packages is 15.66 . We can even improve that, if we allow the system to repeat this planning up to three times. For each repetition, the system sends a new query to the planning server and receives a suggestion that varies. The best result of those three calculations is chosen. Then, the truck can deliver 15.75 packages in average with a positive remaining shelf-life value. Compared with the serverbased approach, we have an improvement of $64.5 \%$ or even $68.6 \%$.

\section{Future research}

In the future, we will continue our research into local pre-processing of sensor data. An important aim is to improve the communication path from the wireless sensor network to the vehicle processor by shifting the task of pre-processing of the sensor data into the sensor network. To do this, sensor nodes with more computation power will be needed. We already started experiments with the Imote2 platform from Crossbow Technology. This platform has an Intel Xscale PXA27x processor with up to $416 \mathrm{MHz}$ and $32 \mathrm{MB}$ RAM. Although these devices are too expensive for practical applications, we want to use them to determine how much calculation power is necessary to fulfil our requirements. It is too difficult to find that out on a platform that has too many restrictions. 
The shift of pre-processing into the sensor network allows a decrease in the number of transmissions inside the wireless sensor network. This saves the energy of the battery-powered devices and hence increases their lifetimes.

Another aspect of our future work is the improvement of the received signal strength inside the sensor network. We noticed problems with damping when the container was loaded because vegetables and fruit have a high percentage of water. Not all the sensor nodes could be reached because the RSSI (received signal strength indicator) values were too bad. Currently, we are developing a multi-hop protocol to transfer the data step-by-step through the network to the gateway, from one sensor node to the next until it reaches the information sink.

We also have a cooperation with Ambient Systems, which is a commercial provider of wireless sensor networks for logistic purposes. Ambient Systems uses a different approach to wireless sensor networks. In addition to distributed sensor nodes and a central gateway, they introduce so-called microrouters, which act as repeaters in the cargo hold to increase the range, so the smartpoints can use less energy to transmit their messages.

\section{Conclusions}

In this paper, we presented our approach to improving food quality by using modern technologies like wireless sensor networks, RFID, software agents, GPS, and mobile communication networks. The local preprocessing of sensor data is an important step for autonomous control. This approach is also a potential way to reduce costs, because the amount of transmitted data becomes significantly lower.

The results of our software simulation show a potential method to optimize the quality of freight items on arrival. An intelligent replanning of the route can save packets that otherwise would run out of quality. This replanning process can be activated by a decision of an agent, which observes the freight's transport conditions.

By benefiting from the interdisciplinary competences of our Collaboration Research Cluster, we will improve our demonstration system and implement our dynamic approach.

\section{Acknowledgements}

This research was supported by the German Research Foundation (DFG) as part of the Collaborative Research Centre 637 "Autonomous Cooperating Logistic Processes".

\section{References}

[1] "The intelligent container project," http://www.intelligentcontainer.com.

[2] S. Arrhenius, "Über die Reaktionsgeschwindigkeit bei der Inversion von Rohrzucker durch Säuren," Zeitschrift für physikalische Chemie, vol. 4, 1889.

[3] L. M. M. Tijskens and J. J. Polderdijk, "A generic model for keeping quality of vegetable produce during storage and distribution," Agricultural Systems, vol. 51, pp. 431-452, 1996.

[4] R. E. Schouten, T. P. M. Huijben, L. M. M. Tijskens, and O. v. Kooten, "Managing biological variance," Acta Hort pp. 131-138, 2006.

[5] F. Bellifemine, G. Caire, A. Poggi, and G. Rimassa, "JADE - A white paper," TILAB EXP in Search of Innovation, Italy, vol. 3, 2003.

[6] R. Jedermann, C. Behrens, D. Westphal, and W. Lang, "Applying autonomous sensor systems in logistics combining sensor networks, RFIDs and software agents," Sensors and Actuators A: Physical, vol. 132, pp. 370-375, 8. Nov. 2006.

[7] R. Jedermann and W. Lang, "The benefits of embedded intelligence - Tasks and applications for ubiquitous computing in logistics," in IOT 2008, LCNS 4952, C. Floerkemeier, Ed. Berlin Heidelberg: Springer-Verlag, 2008, pp. 105-122. 